\title{
Constructing hydrophobic protection for ionic interactions toward water, acid, and base-resistant self-healing elastomers and electronic devices
}

\author{
Linjun Zhang, Hui Xiong, Qi Wu, Yan Peng, Yong Zhu, Hao Wang, Yi Yang, Xikui Liu, \\ Guangsu Huang and Jinrong $\mathrm{Wu}^{*}$
}

\begin{abstract}
Dynamically crosslinked materials generally lose their self-healing ability and mechanical robustness in aqueous, acidic, and basic environments due to disruption of their dynamic interactions and bonds. Herein, a micelle-like structure with a hydrophobic outer layer is used to protect ionic interactions. This structure ensures the self-healing and long-term stability of the ionically crosslinked elastomers in aqueous, acidic, and basic environments. The elastomer possesses a tensile strength of 6.7 MPa and a strain at break of $1400 \%$, which is superior to the existing waterproof selfhealing elastomers. The strain sensors and dielectric actuators based on the elastomer are highly stable and self-healable, even in extremely harsh environments. This design strategy of hydrophobic protection for dynamic interactions is quite general, allowing it to be extended to other self-healing materials.
\end{abstract}

Keywords: underwater self-healing, acid and base resistance, ionic interactions, micelle-like structure, electronic devices

\section{INTRODUCTION}

Self-healing elastomers are smart materials [1] that hold great potential for improving the service life and reliability of vehicles [2], spacecraft [3], batteries [4,5], soft robotics [6,7], and wearable devices [8,9]. To date, various self-healing elastomers have been developed based on supramolecular interactions [10-14] or dynamic covalent bonds [15-18]; however, the majority of self-healing elastomers are susceptible to humidity and water, which strongly deteriorates their mechanical properties and selfhealing ability. Strongly acidic or basic conditions are particularly problematic [19] because most supramolecular interactions and dynamic covalent bonds are unstable and thus easily dissociate under these conditions
[20]. Therefore, method to reasonably design self-healing elastomers that are tolerant to complicated conditions is an important challenge.

The first underwater self-healing materials were developed based on boronic acid derivatives (boronic esters) [21,22] and hydrogen bonds [23]. For example, a selfhealing polyurethane with reversible catechol-boronic ester bonds remained stable underwater for a certain time [24]. A polydimethylsiloxane polymer relying on multistrength hydrogen bonding interactions exhibited underwater self-healing [25]. However, boronic acid derivatives tend to hydrolyze, and their hydrogen bonds are readily saturated by water molecules; meanwhile, they are sensitive to acid and alkaline environments. The existence of polar atoms on the main chains results in the swelling of polymers by water during long-term applications, leading to reduced mechanical properties. In this context, embedding dynamic bonds in hydrophobic polymers may provide better water resistance $[26,27]$. For example, a hydrophobic fluorinated polymer remained stable underwater and even self-healed in acidic and basic media due to dipole-dipole interactions between the highly polar yet hydrophobic C-F bonds [28]. Polydimethylsiloxane polymers, with the synergistic effects of hydrogen bonding and disulfide metathesis, can also self-heal in acidic and basic media [29]. Regrettably, the tensile strength of these materials is about $0.2 \mathrm{MPa}$, which is much lower than that of conventional elastomers. Recently, hydrophobic polybutadiene segments have been utilized as the soft segments to fabricate urea-urethane materials containing disulfide bonds and hydrogen bonds, endowing materials with underwater self-healing ability and acceptable mechanical properties [30]. Nevertheless, poly-

State Key Laboratory of Polymer Materials Engineering, College of Polymer Science and Engineering, Sichuan University, Chengdu 610065, China * Corresponding author (email: wujinrong@scu.edu.cn) 
urethane materials easily degrade under acidic and alkaline conditions. Accordingly, overcoming this limitation requires the development of a more general strategy to protect dynamic bonds.

Herein, we design a micelle-like structure with a hydrophobic layer to protect the ionic bonds of ionomers (Scheme 1a). Such a structure is obtained by grafting tertbutyl pyridine (TB) onto the chains of bromide butyl rubber (BIIR). The hydrophobic tert-butyl groups are preferentially assembled on the surface of ionic aggregates to form a protective layer. This protection layer, as well as the hydrophobic matrix, prevents pyridyl and bromide ions from the interference of water molecules and $\mathrm{H}^{+}$and $\mathrm{OH}^{-}$ions. As a result, the ionomer displays excellent water, acid, and alkaline resistance, and can completely self-heal under harsh environmental conditions (Scheme 1b). Moreover, the ionomer shows better mechanical properties than most underwater self-healing elastomers. Harnessing these advantages, flexible strain sensors and dielectric actuators fabricated based on the ionomer are highly stable and healable, irrespective of the harsh environment, and thus display great potential in practical applications.

\section{EXPERIMENTAL SECTION}

\section{Preparation of ionomers}

BIIR was mixed with either pyridine (PD) or $\mathrm{TB}$ in a
Huckleberry mill at room temperature, followed by hotpressing at $140^{\circ} \mathrm{C}$ for $15 \mathrm{~min}$. The corresponding products were named PDR and TBR, respectively.

\section{Characterization}

Fourier transform infrared spectroscopy (FTIR) was recorded using a Thermo Scientific Nicolet iS50 FTIR in attenuated total reflection mode. The light transmittance of materials was measured on a SHIMADZU UV-2600 ultraviolet spectrophotometer. Transmission electron microscopy (TEM) experiments were performed on a Tecnai G2F20S-TWIN TEM. The surface morphology of small molecular particles and the self-healing behavior of ionomers in water, acidic, or basic solution were observed using a scanning electron microscope (SEM, Nova nanoSEM450). The elemental contents inside and on the surface of particles were determined by energy disperse spectroscopy (EDS, INCA250 Oxford Instruments, UK). The heat flow curves were recorded on a Q2000 (TA instrument) and the samples were heated from 25 to $150^{\circ} \mathrm{C}$ at the heating rate of $10^{\circ} \mathrm{C} \mathrm{min}^{-1}$. Thermal gravimetric analysis (TGA) curves were measured using a TA Q500 by heating the samples from 25 to $600^{\circ} \mathrm{C}$. Dynamic mechanical properties of the samples were measured on a Q800 (TA Instrument) in tension mode. Water contact angles were measured using a drop shape analyzer (DSA 100, KRUSS, Germany). Tensile experiments were performed on an Instron 5967 tensile tester. Electrochemical

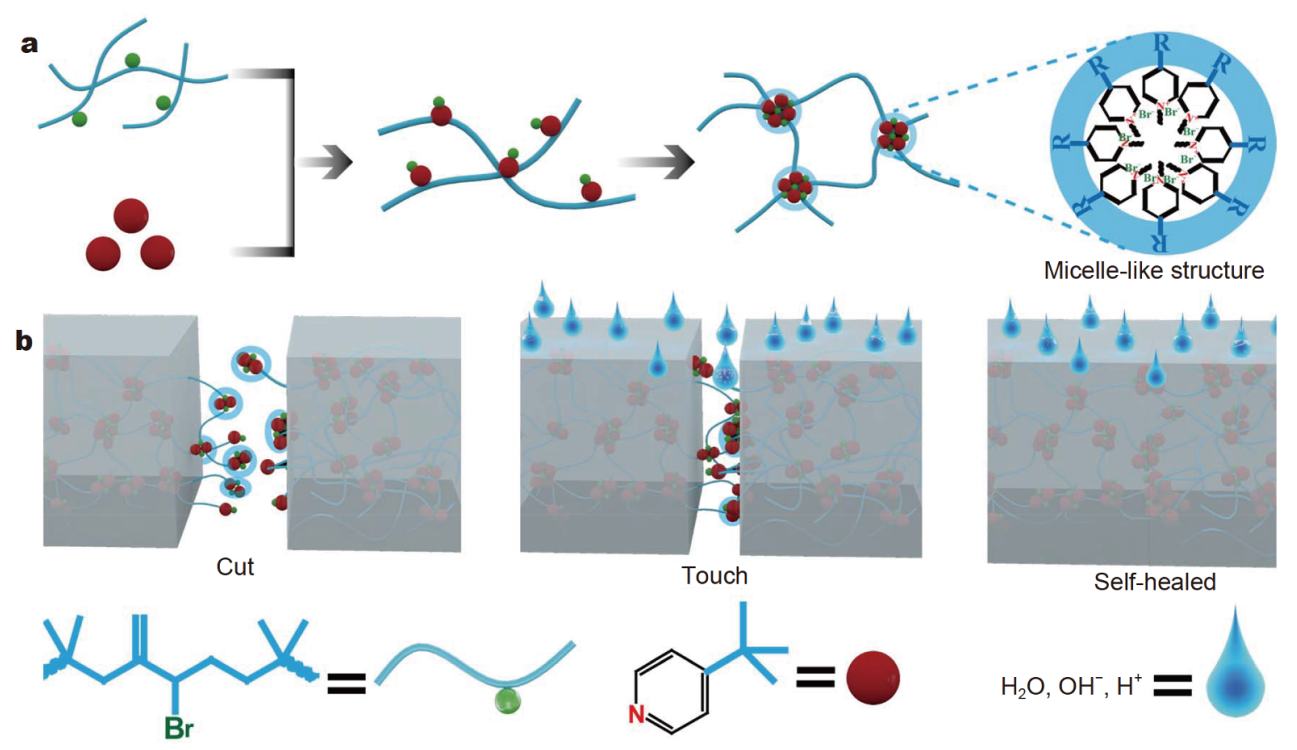

Scheme 1 The design concept of alkyl protection layer for dynamic ionic interactions. (a) Schematic diagram of the synthesis process and the formed micelle-like structure via ionic aggregation. The blue circle represents the hydrophobic molecular layer and $\mathrm{R}$ is the tert-butyl group. (b) Self-healing process in aqueous, acidic, and basic environments. After the fracture interfaces contact, the spontaneous healing of the material is not affected by water molecules, $\mathrm{H}^{+}$, or $\mathrm{OH}^{-}$ions. 
tests were measured on a potentiostat (PGSTAT 302W) using $3.5 \% \mathrm{NaCl}$ electrolyte solution. The thickness of the film coated on the steel was about $0.06 \mathrm{~mm}$. Dielectric measurements were carried out on a Novocontrol Concept 50 system with an Alpha impedance analyzer and a Quatro Cryosystem temperature controller. Electromechanical tensile measurements were performed on a Keithley 6487 in resistance mode assisted with a tensile tester (SANS CMT 4503). The strain measurements of dielectric elastomers were carried out at high-voltage power (TCM600P30-30).

\section{RESULTS AND DISCUSSION}

Constructing hydrophobic protection for polar ionic bonds For general ionomers, polar ionic bonds tend to interact with water molecules, which destroy the dynamic ionic network. To address this issue, a hydrophobic layer was designed to prevent the hydrophilic ionic bonds from interacting with water molecules. To construct such a structure, TB reacted with allyl bromide to form ionic bonds. Due to the co-existence of the non-polar tert-butyl groups and the polar pyridine groups, we envision that TB can self-assemble into a micelle-like structure in a non-polar matrix. As a control, PD without a bulky hydrophobic group can also react with allyl bromide to form ionic bonds. To demonstrate this idea, the water solubility of $\mathrm{PD}$ and $\mathrm{TB}$ was first examined.

$\mathrm{PD}$ is highly soluble in water due to hydrogen bonding interactions between the nitrogen atom and water molecules, leading to a uniformly transparent solution. In sharp contrast, TB with a tert-butyl group stratifies in a static state or forms oil droplets dispersed in water after stirring (Fig. 1a). This phenomenon indicates that the existence of tert-butyl groups significantly reduces the hydrophilicity of PD. To mimic the ionic bonds in BIIR,
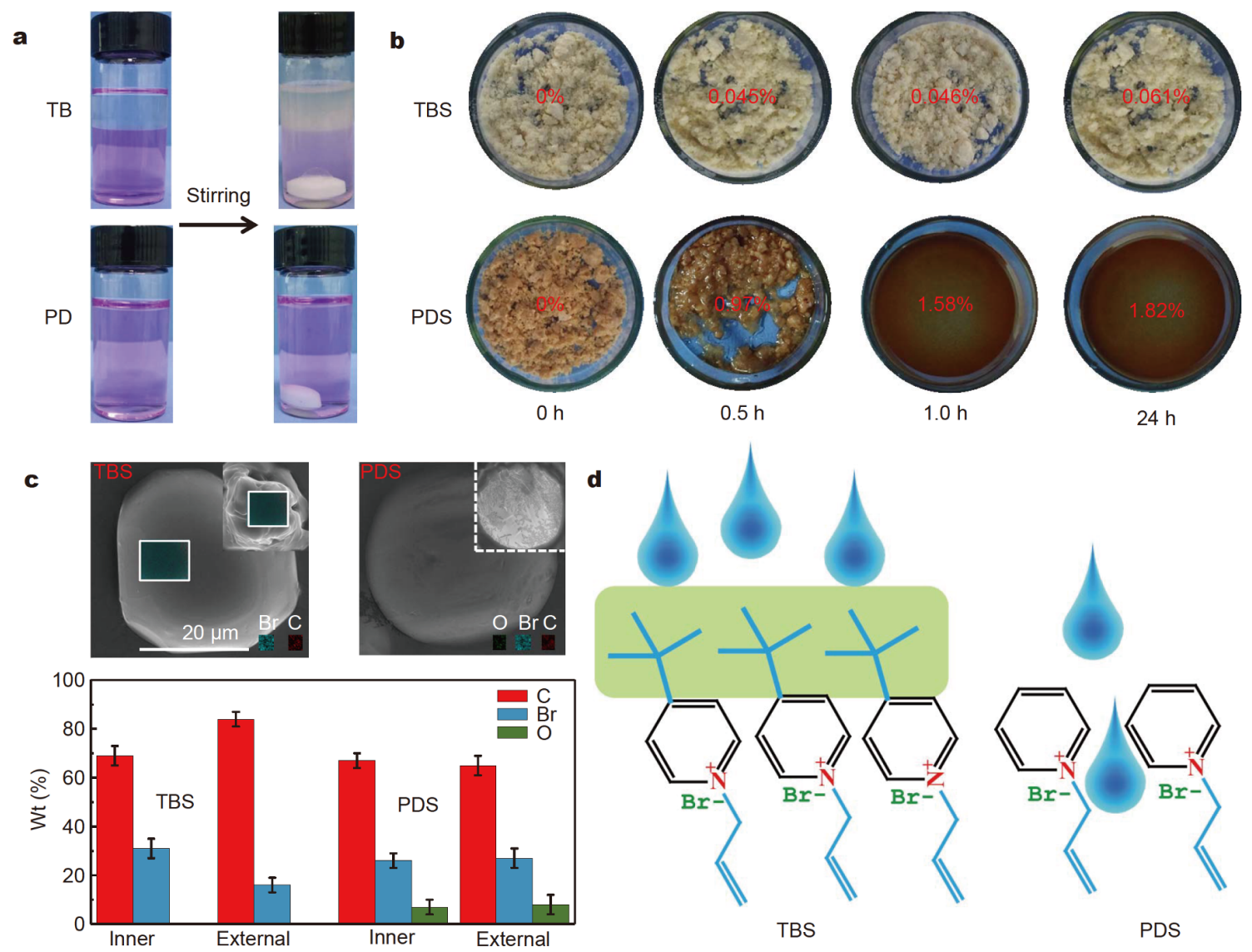

TBS

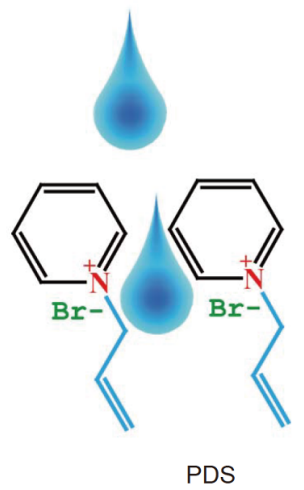

Figure 1 The model experiments of small molecules. (a) The solubility of TB and PD in aqueous solution with a pink dye. (b) Deliquescence process of TBS and PDS in air with 40\% humidity. (c) Characterization of the carbon, bromine, and oxygen contents on the inner and external surface of TBS and PDS particles by SEM and EDS. The inset picture is the fracture surface of broken particles. (d) Schematic diagrams of TBS and PDS being attacked by water molecules. 
$\mathrm{PD}$ and TB reacted with allyl bromide in anhydrous tetrahydrofuran (THF) at $60^{\circ} \mathrm{C}$ for $2 \mathrm{~h} \mathrm{[31],} \mathrm{resulting} \mathrm{in} \mathrm{the}$ corresponding salts, named as PDS and TBS, respectively (Fig. S1). Upon exposure to air with $40 \%$ humidity, TBS only absorbs $0.046 \%$ water within $1 \mathrm{~h}$ at room temperature and remains as a powder for $24 \mathrm{~h}$ (Fig. 1b). In contrast, PDS quickly absorbs $1.58 \%$ water in $1 \mathrm{~h}$, which is about 34-fold higher than that of TBS, and it turns into a reddish-brown liquid.

To explore the underlying reason for this discrepancy, SEM and EDS were used to examine PDS and TBS particles prepared by precipitation in $n$-hexane, which was used to mimic the non-polar matrix of BIIR. SEM images show that the PDS and TBS particles have a diameter range of 10 to $40 \mu \mathrm{m}$. Since EDS cannot measure nitrogen precisely, we use bromine, which usually combines with pyridine rings to represent the hydrophilic content. EDS reveals that the bromine content in the inner TBS particles is much higher than that on the surface, while carbon is more abundant on the exterior. However, the distributions of bromine and carbon are independent of the positions in the PDS particles. Concurrently, oxygen appears in both the interior and exterior of the PDS particles due to rapid water absorption (Fig. 1c). These results reveal that in a nonpolar matrix, the hydrophilic polar ions are concentrated in the interior of the TBR particles, while the hydrophobic carbon groups are more abundant on the exterior, forming a micelle-like structure. This structure can protect the polar ionic bonds and reduce the water absorption rate (Fig. 1d). Nevertheless, PDS without alkyl protection does not form a micelle-like structure; thus, the polar ionic bonds can interact with water molecules, which greatly reduce their stability.

Subsequently, PD and TB were used to crosslink BIIR to form PDR and TBR, respectively. The allylic bromide group of BIIR easily isomerizes from its exo-conformation to endo-conformation, generating reactive species upon heating $[32,33]$. PD and $\mathrm{TB}$ can react with endoallylic bromides to form pyridyl cations and bromide anions in the melt, as shown in Fig. 2a and Fig. S2a. The reaction was monitored by a vulkameter (Fig. $2 \mathrm{~b}$ and Fig. S2b). The result shows that the torque of the ionomer

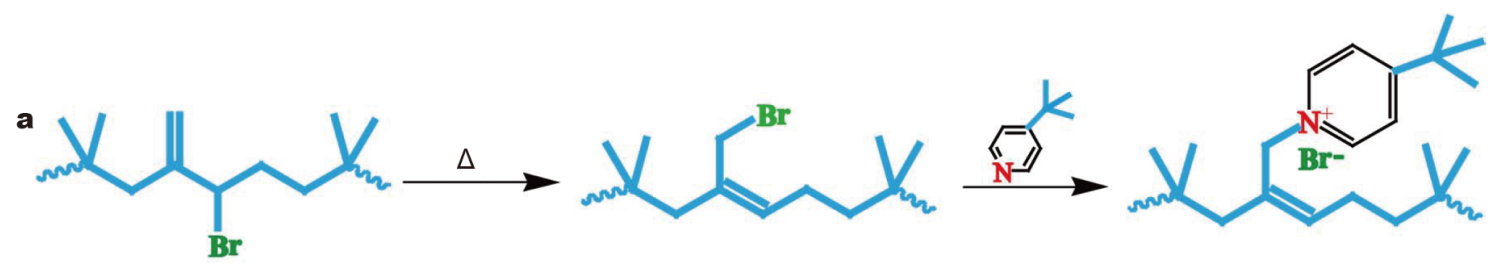

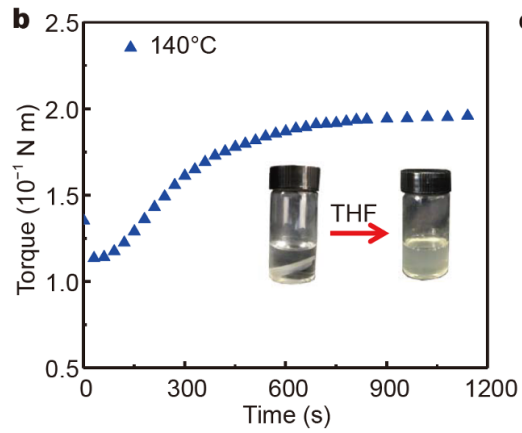

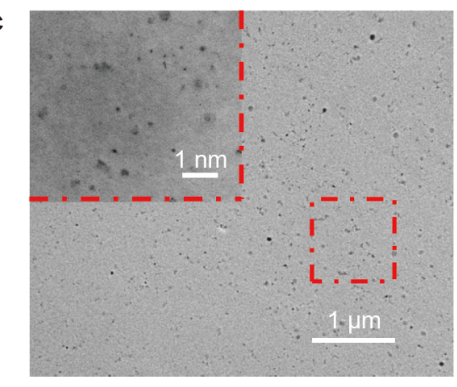

d

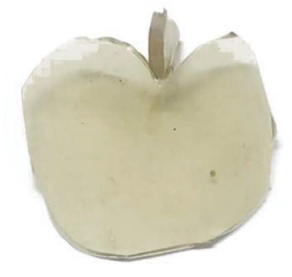

3D apple

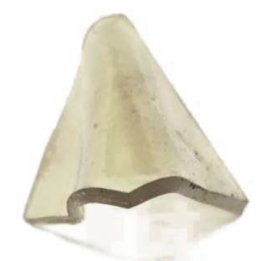

3D aircraft

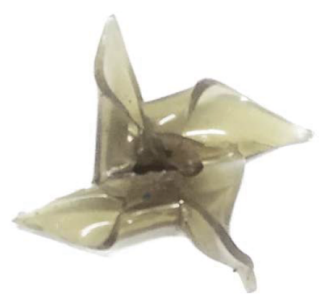

3D Windmill
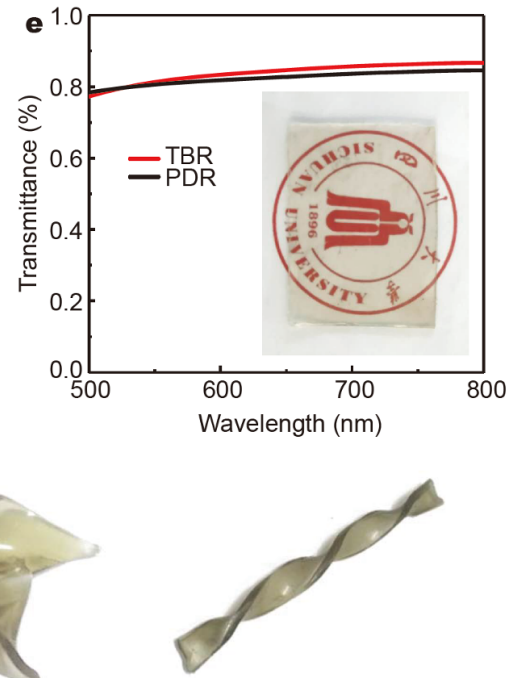

3D helix

Figure 2 Preparation of ionomer materials. (a) The reaction process of TB with bromobutyl rubber. (b) Curing behavior of TBR ionomers at $140^{\circ} \mathrm{C}$. (c) TEM phase diagrams of TBR show distinct ionic aggregates with a diameter range of 8 to $10 \mathrm{~nm}$. (d) Different 3D geometrical shapes including apple, aircraft, windmill, and helix formed via the reconstruction of the ionic network. (e) Transmittance spectra of the PDR and TBR films and a photograph of the film with a thickness of $1.0 \mathrm{~mm}$. 
increases with time, indicating that both $\mathrm{TB}$ and $\mathrm{PD}$ are successfully grafted onto the main chains of BIIR to form an ionic network. The grafting efficiencies of PDR and TBR are $73 \%$ and $84 \%$, respectively, as revealed by ${ }^{1} \mathrm{H}$ NMR in Fig. S3. After the grafting reaction, ionic bonds are formed between the pyridyl and bromide groups [34], which further aggregate to form an ionic network (Fig 2c). Despite the existence of the ionic network, the ionomers are soluble in low-polarity solvents (like THF), demonstrating that the crosslinked structure is of a physical nature. Such a physical network is capable of dynamic exchange and reconstruction via molecular motion (Fig. S2c). Thus, if the materials are configured into various $3 \mathrm{D}$ shapes at high temperatures, these $3 \mathrm{D}$ shapes can be fixed after cooling to room temperature (Fig. 2d). Relying on this feature, flexible devices with complicated shapes can be prepared. In addition, the average transmittance of the ionomers under visible light
(500 to $800 \mathrm{~nm}$ ) is higher than $83 \%$, showing good transparency (Fig. 2e).

To examine the stability of the ionomers under hydrophobic protection, PDR, TBR, and pristine BIIR were immersed in deuterated water $\left(\mathrm{D}_{2} \mathrm{O}\right)$ for $3 \mathrm{~d}$. After immersion, the characteristic peak of $\mathrm{D}_{2} \mathrm{O}$ in the FTIR spectrum is easily recognized at around $2500 \mathrm{~cm}^{-1}$ for PDR [35] and then slowly vanishes when heated at $100^{\circ} \mathrm{C}$ (Fig. 3a). However, there are no signals of $\mathrm{D}_{2} \mathrm{O}$ in the FTIR spectra of TBR and BIIR after immersion. Thermal analyses also confirm this point. A new endothermic peak near $106^{\circ} \mathrm{C}$ appears in the differential scanning calorimetry (DSC) curves for the water-immersed PDR, ascribed to the evaporation of water (Fig. S4a). TGA curves show that the water absorption is about $0.31 \%$ (Fig. S4b, d). The absorbed water in PDR changes the surface tension, resulting in a reduction in the water contact angle with increasing environmental humidity (Fig. 3b).
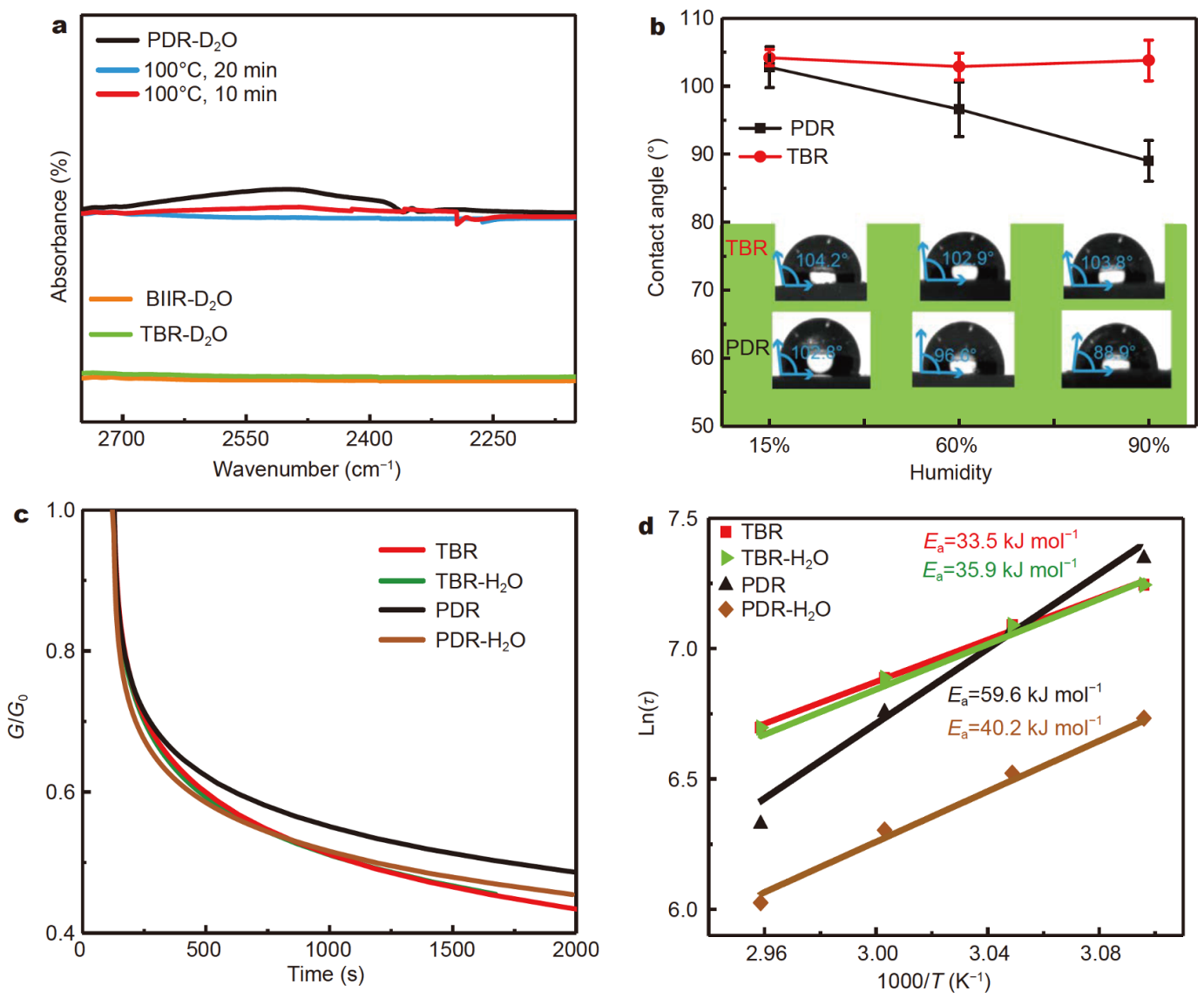

Figure 3 Demonstration of the structural stability and properties under the protection of hydrophobic molecular layers. (a) The FTIR spectra of the $\mathrm{D}_{2} \mathrm{O}$-treated samples. The disappearance of $\mathrm{D}_{2} \mathrm{O}$ in PDR treated at $100^{\circ} \mathrm{C}$ is also shown. (b) The variation in water contact angles of PDR and TBR after treatment in different humidity environments for $3 \mathrm{~d}$. (c) The stress relaxation curves of the original samples and after immersion at $25^{\circ} \mathrm{C}$. (d) Comparison of relaxation activation energies between the original samples and treated samples. 
Nevertheless, there is no weight loss from 100 to $110^{\circ} \mathrm{C}$ for the water-immersed TBR (Fig. S4c, d), and the water contact angle remains constant in environments with different humidities. These results illustrate that although the BIIR matrix is highly hydrophobic, PDR is still susceptible to water due to the absence of the micelle-like structure [36], while TBR is highly water-proof due to the protection of the hydrophobic layer formed by tert-butyl groups.

To analyze the influence of water molecules on the network dynamics, stress relaxation tests were performed by applying a constant strain to the samples. Notably, the pristine TBR and water-immersed TBR show nearly identical stress relaxation curves at $25^{\circ} \mathrm{C}$, and the same relaxation activation energy calculated by Arrhenius fitting (Fig. 3c, d). Therefore, water immersion has a negligible influence on the network dynamics of TBR. However, immersion distinctly increases the relaxation speed of PDR (Fig. 3c), suggesting faster network dynamics due to the plasticizing effect of water [37,38]. As a result, the relaxation activation energy decreases from $59.6 \mathrm{~kJ} \mathrm{~mol}^{-1}$ of pristine PDR to $40.2 \mathrm{~kJ} \mathrm{~mol}^{-1}$ of the immersed PDR (Fig. 3d). This phenomenon reveals that the water molecules partially destroy the ionic interactions and weaken the binding energy of PDR (Fig. S4e).

\section{Water insensitivity and acid-based tolerance}

Pristine TBR shows high mechanical properties, with a tensile strength of $6.7 \mathrm{MPa}$ and a strain at break of $1400 \%$, which are $34 \%$ and $12 \%$ higher than those of pristine PDR, respectively, as shown in Fig. 4a. The higher mechanical properties are closely related to the electron-donating effect of the tert-butyl group of TB. ${ }^{1} \mathrm{H}$ NMR spectra indicates that the $\mathrm{H}^{1}$ adjacent to the nitrogen of the pyridine ring shifts downfield after the grafting reaction (Fig. S3). The chemical shift of TBR is much higher than that of PDR, due to the stronger electron-donating effect of the tert-butyl group of TB. The greater chemical shift qualitatively reflects enhanced interactions between the pyridyl cation and bromine anion of TBR [40]. Consequently, TBR shows greater mechanical properties than PDR. Moreover, the mechanical properties of TBR are higher than those of most waterproof self-healing elastomers (Fig. 4b).

To examine the underwater stability, the mechanical properties of the ionomers after immersion in water for $3 \mathrm{~d}$ were also measured. The tensile strength of the waterimmersed PDR decreases to $3.0 \mathrm{MPa}$, which is much lower than the $5.1 \mathrm{MPa}$ of the pristine PDR (Fig. 4a). This reduction in the tensile strength $(42 \%)$ is almost identical to that of the relaxation activation energy of PDR (43\%). Simultaneously, the strain at break increases, and the strain hardening becomes weaker for the water-immersed PDR. Such changes in mechanical properties show that the water molecules can disrupt the ionic network of PDR. In contrast, the ionic interactions of TBR are quite stable, and its mechanical performance is hardly affected by water. After the soaking time is prolonged to three months, the tensile strength even becomes slightly higher than that of the original TBR (Fig. S5a), but PDR becomes very weak, with a tensile strength of around $0.8 \mathrm{MPa}$ (Fig. S5b).

Elastomers are usually applied as anti-corrosion coatings for metals used in acidic or basic conditions. To evaluate the acid and base resistance of the ionomers, their tensile strength retention rates were measured. After soaking in highly acidic ( $\mathrm{pH} 2)$ or alkaline ( $\mathrm{pH} 14)$ solutions for $3 \mathrm{~d}$, the tensile strength of TBR is almost unchanged. In sharp contrast, the tensile strength retention rate of PDR is only about $60 \%$ (Fig. 4c). Tafel polarization tests of samples with thicknesses of $0.06 \mathrm{~mm}$ on a metal surface treated in acidic and alkaline solutions for $3 \mathrm{~d}$ were performed in $3.5 \% \mathrm{NaCl}$ electrolyte. The position of the Tafel curves of TBR is independent of the treatment conditions (Fig. 4d), and the nearly equal corrosion voltage $E_{\text {corr }}$ calculated by extrapolation between the pristine TBR and treated TBR illustrates the excellent corrosion resistance (Fig. 4f). In contrast, the Tafel curves of the treated PDR significantly shift to left compared with that of the original PDR (Fig. 4e), leading to a reduced $E_{\text {corr }}$ (Fig. $4 \mathrm{f}$ ). These results demonstrate the better corrosion resistance of TBR than PDR. Moreover, combining good binding property with acid and base tolerance, an ultrathin protective TBR membrane (thickness $\sim 200 \mu \mathrm{m}$ ) was coated on $\mathrm{pH}$ paper to further verify the corrosion resistance under harsh conditions. The color of the $\mathrm{pH}$ paper coated with TBR does not change after treatment in acidic and basic solutions for one week at room temperature, or even after being heated at $60^{\circ} \mathrm{C}$ for $3 \mathrm{~d}$ (Fig. $4 \mathrm{~g}$ ). These results demonstrate the very high stability and reliability of TBR under acidic and alkaline conditions, due to the hydrophobic tert-butyl layer, as well as the hydrophobic BIIR molecules. Collectively, the TBR ionomer is superior to most self-healing elastomers in terms of its water insensitivity, acidbase tolerance and mechanical properties (Fig. 4b).

\section{Self-healing behavior in harsh environments}

Apart from the excellent mechanical properties and stability in aqueous, acidic, and alkaline conditions, TBR 

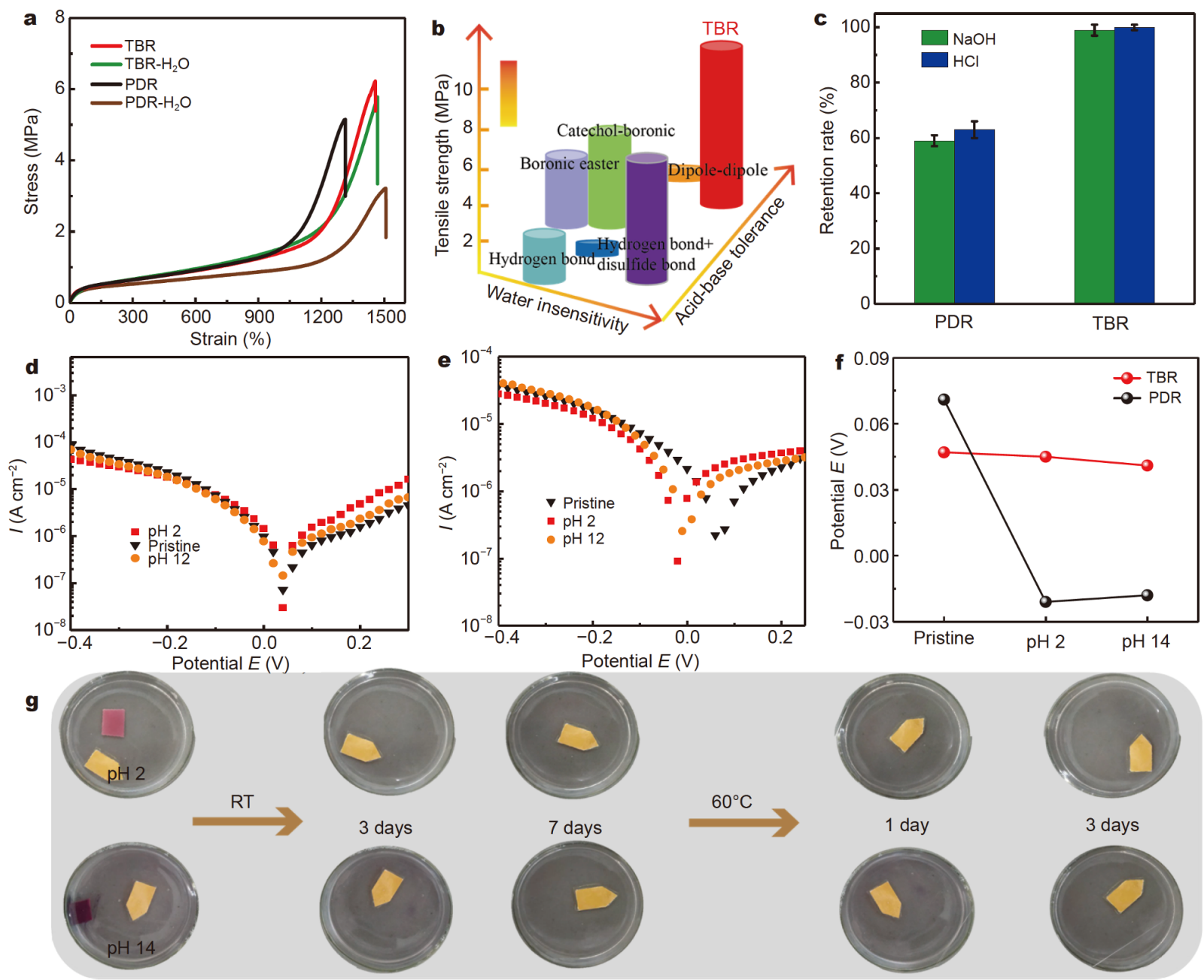

Figure 4 Water insensitivity and acid-base tolerance. (a) The tensile curves of the original samples and those after immersion in water for $3 \mathrm{~d}$. (b) Ashby diagram comparing the tensile strength, acid, and base resistance of TBR with those of other waterproof self-healing elastomers based on hydrogen bonds [25], disulfide bonds [27,29,30], dipole-dipole [28,39], boron ester [24], and catechol-boronic bonds [19,22]. (c) Retention rates of the tensile strengths of TBR and PDR after immersion in acidic and basic solutions for $3 \mathrm{~d}$. Tafel polarization curves of (d) TBR and (e) PDR. (f) The corrosion voltage $E_{\text {corr }}$ obtained by Tafel curve extrapolation. $(\mathrm{g})$ The $\mathrm{pH}$ test paper coated with the protective TBR film was immersed in acidic and basic solutions. The thickness of the film is around $200 \mu \mathrm{m}$.

also reveals an outstanding self-healing capability because of dynamic ionic interactions. The dynamic ionic groups can exchange between ionic clusters, giving rise to the reconfiguration of the ionic network and the recovery of mechanical properties [41]. In dry air at $60^{\circ} \mathrm{C}$, TBR exhibits a self-healing efficiency of about $100 \%$, which is defined as the ratio between the recovered tensile strength and the pristine tensile strength (Fig. S6a). Relying on the protection of the hydrophobic effect, TBR is also able to self-heal underwater. To demonstrate this behavior, a surface-scratch-recovery test was performed. A scratch with a width of about $30 \mu \mathrm{m}$ was created by a blade on the TBR sample. The SEM images display that the scratch is gradually repaired in water at $60^{\circ} \mathrm{C}$, and after $18 \mathrm{~h}$, the wound almost completely vanishes (Fig. 5a). Moreover, the TBR samples were cut into halves, then gently put together, and then immediately left in water to allow healing. The completely fractured samples could still selfheal underwater (Fig. 5b and Movie S1). Unlike most selfhealing polymers, their fractured halves gradually lost adhesion on the re-joined interface and were then totally separated by the water molecules. The fractured TBR samples exhibited fairly good underwater adhesion, even when stirred (Fig. S6b and Movie S2). The underwater self-healing was allowed to proceed for $18 \mathrm{~h}$ at different temperatures. With increasing temperature, the under- 
a

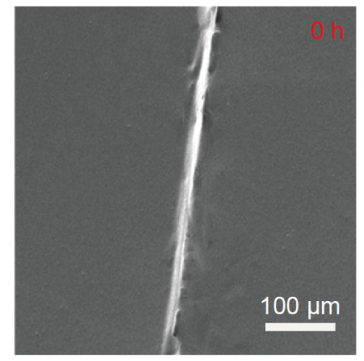

b
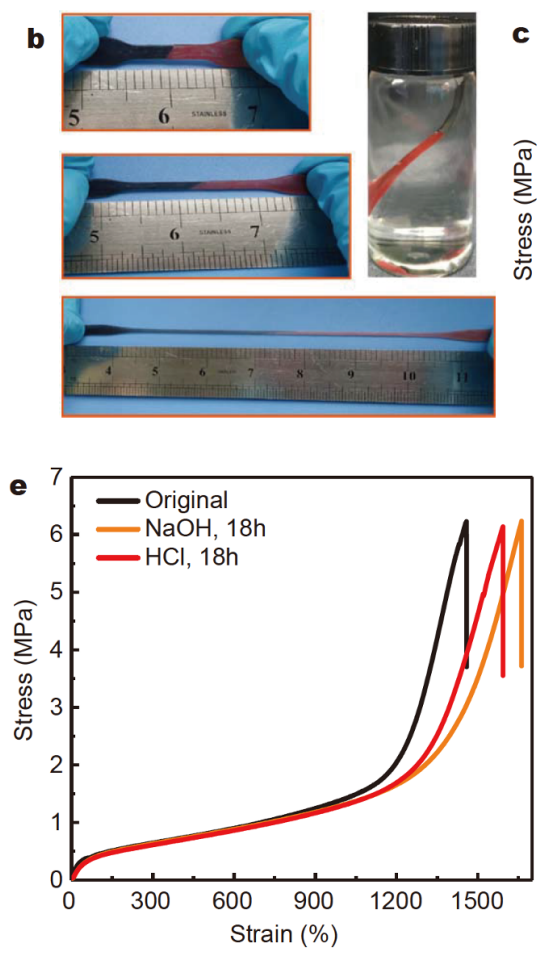
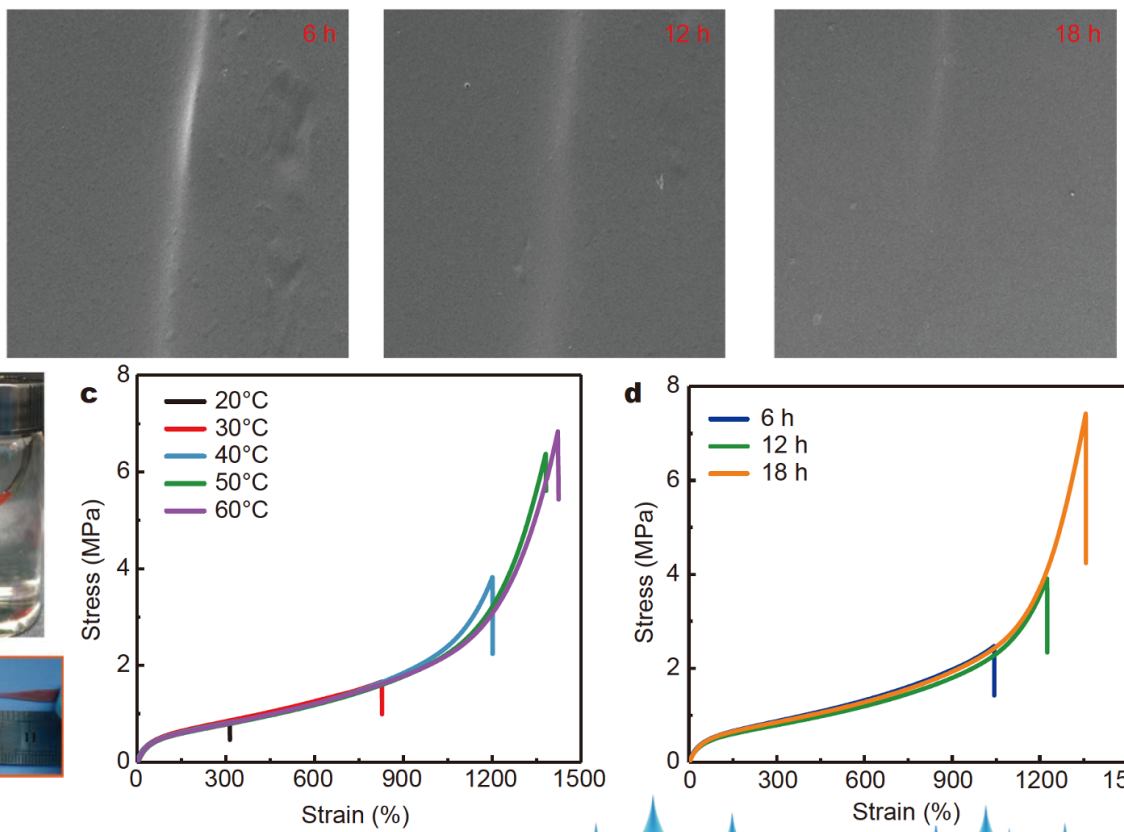

f
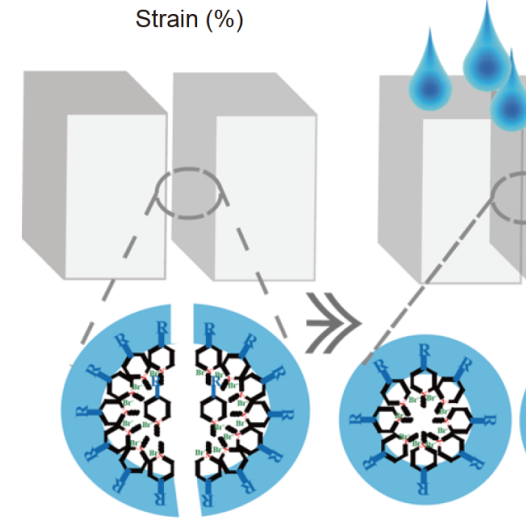

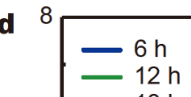

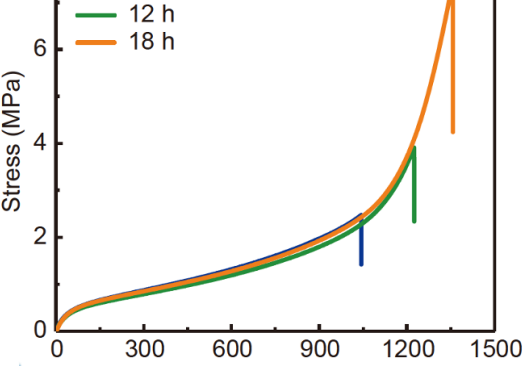

Strain (\%)

Figure 5 The self-healing behavior in harsh environments. (a) SEM images of underwater self-healing behavior of the TBR film with a thickness of $1 \mathrm{~mm}$ after being scratched. The scratch is entirely healed in $60^{\circ} \mathrm{C}$ water after $18 \mathrm{~h}$. (b) Photographs of the underwater self-healing process. The two parts with different colors were spliced together and healed in $60^{\circ} \mathrm{C}$ water for $18 \mathrm{~h}$. The two parts were completely rejoined. (c) Typical stress-strain curves of repaired TBR samples immersed in water at different temperatures for $18 \mathrm{~h}$. (d) Typical stress-strain curves of repaired TBR samples under $60^{\circ} \mathrm{C}$ water for various times. (e) Representative stress-strain curves of TBR after self-healing in $60^{\circ} \mathrm{C}$ acidic and basic solutions. (f) The schematic diagram of the underwater self-healing mechanism.

water self-healing efficiency gradually increases (Fig. 5c) due to the enhanced chain mobility and exchange rate of ions between ionic clusters at high temperatures. As a result, TBR recovers nearly $100 \%$ of its initial tensile strength at $60^{\circ} \mathrm{C}$ after $18 \mathrm{~h}$ underwater (Fig. 5d). This time is in line with that of the surface scratch tracked by SEM, which reveals the consistency of the internal and surface healing behavior underwater.

In harsh environments, such as strongly acidic and alkaline conditions, materials should be able to self-heal, as the materials are easily damaged and manual repairs are extremely difficult in these environments. The surface-scratch-recovery tests show that the scratch on TBR gradually vanishes, even in the environments where $\mathrm{pH}$ is 2 or 14 (Fig. S7a).

In these conditions, the completely fractured TBR samples also show a self-healing efficiency of nearly $100 \%$ at $60^{\circ} \mathrm{C}$ (Fig. 5e and Fig. S7b). These results show that self-healing is unaffected by $\mathrm{H}^{+}$and $\mathrm{OH}^{-}$ions.

The self-healing mechanism underwater and in acidic and alkaline conditions is shown in Fig. 5f. The broken ionic aggregates on the fresh interfaces are easily reconstructed to form a new micelle-like structure via local rearrangement of polar ions [42]; this makes the polar ions again wrapped by the hydrophobic molecular layers, which protects the hydrophilic ionic bonds. This me- 
chanism can be demonstrated by two phenomena. One is that the fresh section of TBR displays nearly the same water contact angle as that of the normal surface (Fig. S8a), suggesting that the surface tension of the fresh section does not change, even when the ionic aggregates are fractured. The other is that TBR can accomplish a nearly $100 \%$ self-healing efficiency, even if the fresh sections are exposed to air with $40 \%$ humidity for $24 \mathrm{~h}$, suggesting that the broken ionic aggregates are isolated from the environment (Fig. S8b).

Electronic devices for harsh environments

By virtue of the inherent advantages of TBR, a new type of water, acid and base-resistant strain sensor was developed. The strain sensor is composed of a sandwich structure with one layer of carbon nanotubes (CNT) sandwiched between two TBR sheets. The SEM image reveals good interfacial fusion of the sandwich structure (Fig. 6a). The strain sensor displays increasing resistance with strain during stretching (Fig. 6b and Fig. S9a). Such a strain response can be used to monitor different types of strain motions. Moreover, the output signal of the strain sensor is exceedingly similar and stable in various environments, such as basic conditions (Fig. 6c, d). These properties are verified by bending motion of fingers underwater (Fig. 6e) and linear stretching of the strain

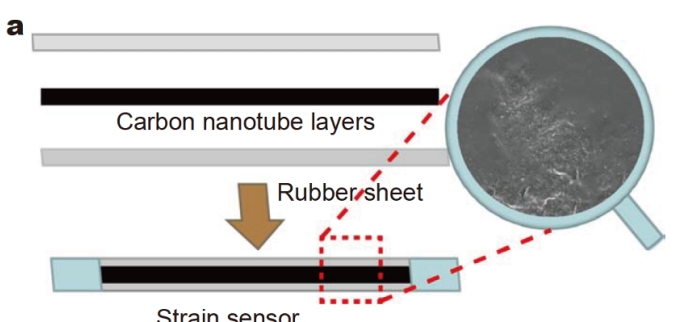

Strain sensor

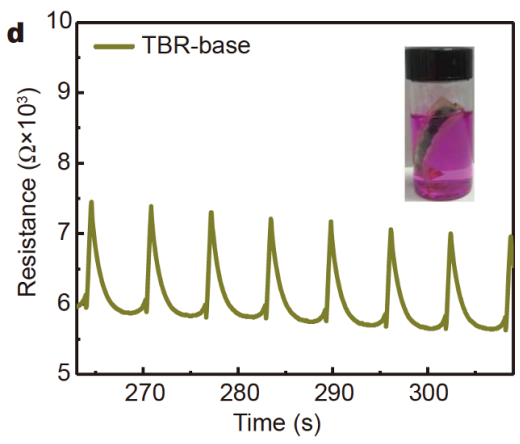

h

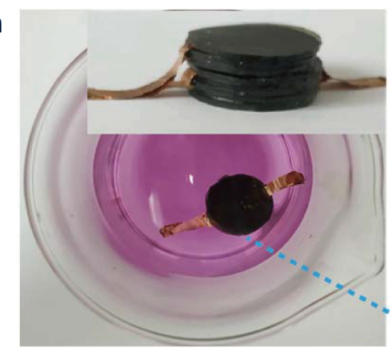

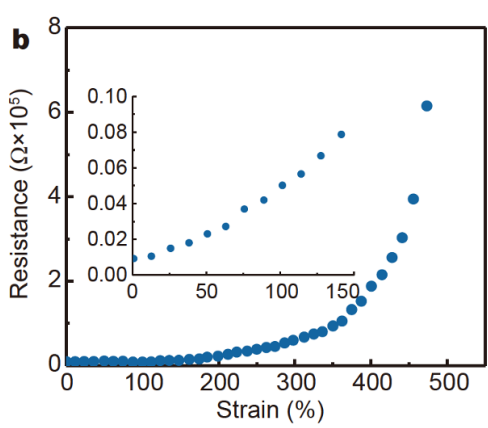

e
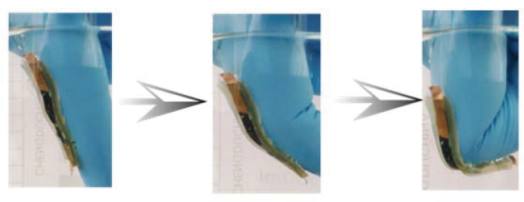

f
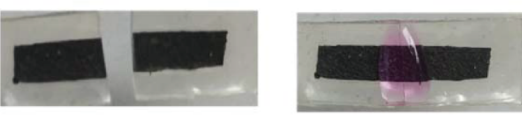

i

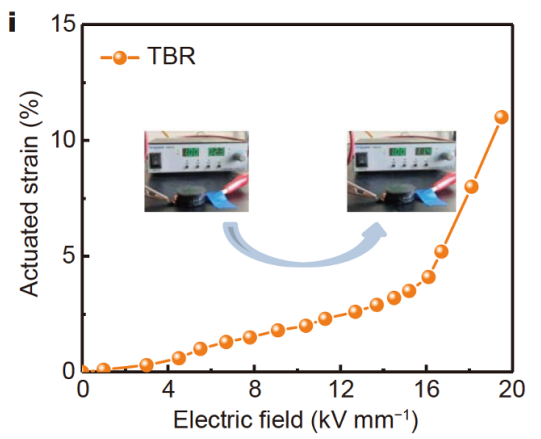

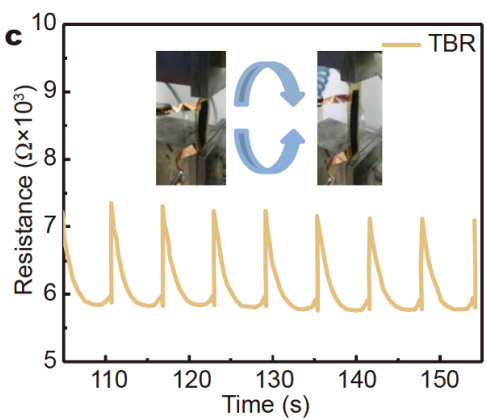
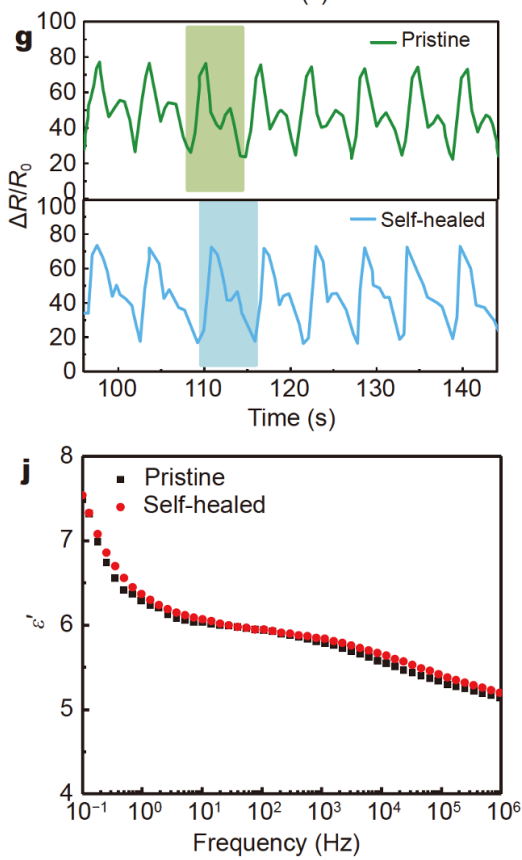

Figure 6 Demonstration of soft devices in harsh environments. (a) The sketch map of a sandwich-shaped stain sensor, which consists of two rubber sheets and a carbon nanotube layer that is laminated between two rubber sheets. The ends of the sensor are the electrode made from conductive copper foil. The SEM image is the section of the strain sensor. (b) Resistance-strain curve of the strain sensor. Comparison of the resistance signals between (c) the original sensor of TBR and (d) the sensor after treatment in basic solution for one week. (e) Detection of strain signal of the bending movement of fingers. (f) The photographs of the cut-off strain sensor healed in a basic solution at $60^{\circ} \mathrm{C}$ for $18 \mathrm{~h}$. (g) The finger-bending signal of the original strain sensor and the healed strain sensor. (h) Multilayered (7 layers) dielectric elastomer actuator. (i) The actuated strain of the actuator as a function of the applied voltage. (j) The dielectric constant of the original actuator and that of the cut-off actuator healed in basic solution at $60^{\circ} \mathrm{C}$ for $18 \mathrm{~h}$. 
sensor in a circuit (Fig. S9b), which leads to a relative resistance change and light-emitting diode (LED) brightness change, respectively. The signal shape and the change amplitudes of the relative resistance and LED brightness remain nearly the same after the strain sensors are completely cut off and healed at $60^{\circ} \mathrm{C}$ in alkaline solution for $18 \mathrm{~h}$ (Fig. 6f, g and Movie S3). In addition, a multilayered dielectric elastomer actuator, fabricated by a stacking method (Fig. 6h), can generate large strain when a voltage is applied (Fig. 6i). The dielectric constant of the repaired elastomer in alkaline solution is nearly equal to that of the original elastomer (Fig. 6j), indicating that the dielectric network structure has been entirely recovered. These results demonstrate that the strain sensors and actuators display reliable signal transmission, actuation, and self-healing in several complex environments. Such a feature is critical for the practical application of electronic devices in harsh environments.

\section{CONCLUSIONS}

This work provides a new strategy to protect dynamic ionic interactions by constructing a micelle-like structure with a hydrophobic molecular layer. This hydrophobic layer relies on tert-butyl groups to protect against the interference of water, acid, and base molecules. This endows the material with excellent long-term stability, selfhealing, and mechanical performance. Integrating all these advantages, we developed soft strain sensors and dielectric actuators that hold great promise in applications such as soft robotics and wearable devices. The design concept and synthesis of the micelle-like structure is quite simple, and can thus be utilized to protect other dynamic interactions and bonds, which may pave the way to fabricate robust self-healing materials that can endure diverse harsh environments.

\section{Received 7 August 2020; accepted 3 November 2020;} published online 11 January 2021

1 Chen Y, Kushner AM, Williams GA, et al. Multiphase design of autonomic self-healing thermoplastic elastomers. Nat Chem, 2012, 4: $467-472$

2 Trovatti E, Lacerda TM, Carvalho AJF, et al. Recycling tires? Reversible crosslinking of poly(butadiene). Adv Mater, 2015, 27: 2242-2245

$3 \mathrm{Li} \mathrm{Y}$, Chen $\mathrm{S}, \mathrm{Wu} \mathrm{M}$, et al. Polyelectrolyte multilayers impart healability to highly electrically conductive films. Adv Mater, 2012, 24: 4578-4582

4 Mai W, Yu Q, Han C, et al. Self-healing materials for energystorage devices. Adv Funct Mater, 2020, 30: 1909912

5 Jaumaux P, Liu Q, Zhou D, et al. Deep-eutectic-solvent-based selfhealing polymer electrolyte for safe and long-life lithium-metal batteries. Angew Chem Int Ed, 2020, 59: 9134-9142
6 Cao J, Zhou C, Su G, et al. Arbitrarily 3D configurable hygroscopic robots with a covalent-noncovalent interpenetrating network and self-healing ability. Adv Mater, 2019, 31: 1900042

7 Zhang Y, Ellingford C, Zhang R, et al. Electrical and mechanical self-healing in high-performance dielectric elastomer actuator materials. Adv Funct Mater, 2019, 29: 1808431

8 Huynh TP, Sonar P, Haick H. Advanced materials for use in soft self-healing devices. Adv Mater, 2017, 29: 1604973

9 Khatib M, Zohar O, Saliba W, et al. A multifunctional electronic skin empowered with damage mapping and autonomic acceleration of self-healing in designated locations. Adv Mater, 2020, 32: 2000246

10 Cordier P, Tournilhac F, Soulié-Ziakovic C, et al. Self-healing and thermoreversible rubber from supramolecular assembly. Nature, 2008, 451: 977-980

11 Herbst F, Döhler D, Michael P, et al. Self-healing polymers via supramolecular forces. Macromol Rapid Commun, 2013, 34: 203220

12 Zhang L, Liu Z, Wu X, et al. A highly efficient self-healing elastomer with un-precedented mechanical properties. Adv Mater, 2019, 31: e1901402

13 Zhou X, Wang L, Wei Z, et al. An adaptable tough elastomer with moisture-triggered switchable mechanical and fluorescent properties. Adv Funct Mater, 2019, 29: 1903543

14 Wu J, Cai LH, Weitz DA. Tough self-healing elastomers by molecular enforced integration of covalent and reversible networks. Adv Mater, 2017, 29: 1702616

15 Chen Y, Tang Z, Zhang X, et al. Covalently cross-linked elastomers with self-healing and malleable abilities enabled by boronic ester bonds. ACS Appl Mater Interfaces, 2018, 10: 24224-24231

16 Ying $\mathrm{H}$, Zhang Y, Cheng J. Dynamic urea bond for the design of reversible and self-healing polymers. Nat Commun, 2014, 5: 3218

17 Zou W, Jin B, Wu Y, et al. Light-triggered topological programmability in a dynamic covalent polymer network. Sci Adv, 2020, 6: eaaz2362

18 Wei H, Yang Y, Huang X, et al. Transparent, robust, waterresistant and high-barrier self-healing elastomers reinforced with dynamic supramolecular nanosheets with switchable interfacial connections. J Mater Chem A, 2020, 8: 9013-9020

19 Li J, Ejima H, Yoshie N, et al. Seawater-assisted self-healing of catechol polymers via hydrogen bonding and coordination interactions. ACS Appl Mater Interfaces, 2016, 8: 19047-19053

20 Huynh T, Khatib M, Haick H. Self-healable materials for underwater applications. Adv Mater Technol, 2019, 4: 1900081

21 Deng CC, Brooks WLA, Abboud KA, et al. Boronic acid-based hydrogels undergo self-healing at neutral and acidic pH. ACS Macro Lett, 2015, 4: 220-224

22 Kim C, Ejima H, Yoshie N. Non-swellable self-healing polymer with long-term stability under seawater. RSC Adv, 2017, 7: 1928819295

23 Ahn BK, Lee DW, Israelachvili JN, et al. Surface-initiated selfhealing of polymers in aqueous media. Nat Mater, 2014, 13: 867872

24 Xia NN, Rong MZ, Zhang MQ. Stabilization of catechol-boronic ester bonds for underwater self-healing and recycling of lipophilic bulk polymer in wider ph range. J Mater Chem A, 2016, 4: 1412214131

25 Kang J, Son D, Wang GN, et al. Tough and water-insensitive selfhealing elastomer for robust electronic skin. Adv Mater, 2018, 30: e1706846 
26 Schulte VA, Hu Y, Diez M, et al. A hydrophobic perfluoropolyether elastomer as a patternable biomaterial for cell culture and tissue engineering. Biomaterials, 2010, 31: 8583-8595

27 Ying WB, Yu Z, Kim DH, et al. Waterproof, highly tough, and fast self-healing polyurethane for durable electronic skin. ACS Appl Mater Interfaces, 2020, 12: 11072-11083

28 Cao Y, Wu H, Allec SI, et al. A highly stretchy, transparent elastomer with the capability to automatically self-heal underwater. Adv Mater, 2018, 30: e1804602

29 Guo H, Han Y, Zhao W, et al. Universally autonomous self-healing elastomer with high stretchability. Nat Commun, 2020, 11: 2037

30 Khatib M, Zohar O, Saliba W, et al. Highly efficient and waterinsensitive self-healing elastomer for wet and underwater electronics. Adv Funct Mater, 2020, 30: 1910196

31 Rudine AB, Walter MG, Wamser CC. Reaction of dichloromethane with pyridine derivatives under ambient conditions. J Org Chem, 2010, 75: 4292-4295

32 Suckow M, Mordvinkin A, Roy M, et al. Tuning the properties and self-healing behavior of ionically modified poly(isobutylene-coisoprene) rubber. Macromolecules, 2018, 51: 468-479

33 Das A, Sallat A, Bohme F, et al. Ionic modification turns commercial rubber into a self-healing material. ACS Appl Mater Interfaces, 2015, 7: 20623-20630

34 Mordvinkin A, Suckow M, Böhme F, et al. Hierarchical sticker and sticky chain dynamics in self-healing butyl rubber ionomers. Macromolecules, 2019, 52: 4169-4184

35 Zhu Y, Zhang J, Wu Q, et al. Three-dimensional programmable, reconfigurable, and recyclable biomass soft actuators enabled by designing an inverse opal-mimetic structure with exchangeable interfacial crosslinks. ACS Appl Mater Interfaces, 2020, 12: 1575715764

36 Semenov AN, Rubinstein M. Dynamics of entangled associating polymers with large aggregates. Macromolecules, 2002, 35: 48214837

37 Kutsumizu S, Nagao N, Tadano K, et al. Effects of water sorption on the structure and properties of ethylene ionomers. Macromolecules, 1992, 25: 6829-6835

38 Yano S, Tadano K, Nagao N, et al. Dielectric relaxation studies on water absorption of ethylene ionomers. Macromolecules, 1992, 25: 7168-7171

39 Zhang Y, Li M, Qin B, et al. Highly transparent, underwater selfhealing, and ionic conductive elastomer based on multivalent iondipole interactions. Chem Mater, 2020, 32: 6310-6317

40 Cui J, Nie FM, Yang JX, et al. Novel imidazolium-based poly(ionic liquid)s with different counterions for self-healing. J Mater Chem A, 2017, 5: 25220-25229

41 Nie FM, Cui J, Zhou YF, et al. Molecular-level tuning toward aggregation dynamics of self-healing materials. Macromolecules, 2019, 52: 5289-5297

42 Register RA. Morphology and structure-property relationships in random ionomers: Two foundational articles from Macromolecules. Macromolecules, 2020, 53: 1523-1526

Acknowledgements This work was supported by the National Natural Science Foundation of China (51873110 and 51673120), State Key Laboratory of Polymer Materials Engineering (sklpme2019-2-14) and the Fundamental Research Funds for Central Universities.

Author contributions Zhang L designed and engineered the samples; Zhang $\mathrm{L}$ and Xiong $\mathrm{H}$ performed the experiments; Zhang $\mathrm{L}$ wrote the paper with support from $\mathrm{Wu}$ J. All authors contributed to the general discussion.

Conflict of interest The authors declare no competing financial interests.

Supplementary information Experimental details and supporting data are available in the online version of the paper.

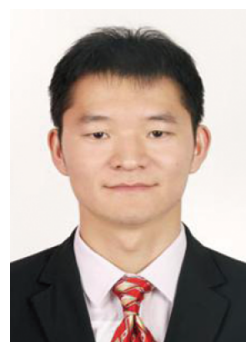

Linjun Zhang received his bachelor's degree from the College of Polymer Science and Engineering at Sichuan University in 2015 and MS degree from the Academy of Aerospace Soild Propulsion Technology in 2018. He is currently pursuing his $\mathrm{PhD}$ at Sichuan University, under the supervision of Prof. Wu. His research interests are focused on the design and preparation of self-healing elastomeric materials and functional elastomeric composites.

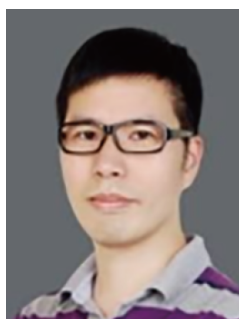

Jinrong $\mathbf{W u}$ is a Professor in the college of Polymer Science and Engineering at Sichuan University. He received his bachelor's degree and doctoral degree in 2003 and 2008 respectively from the College of Polymer Science and Engineering, Sichuan University. He studied at Texas Tech University as a visiting student from 2007 to 2008 and at Harvard University as a visiting scholar from 2014 to 2016 . His current research involves high-performance elastomers, functional elastomers, self-healing elastomers and related theoretical problems of elastomer materials.

\section{基于疏水保护作用的离子相互作用构建耐水和酸 碱的自修复弹性体和电子器件}

张林军, 熊慧, 几琪, 彭燕, 朱勇, 王豪, 杨毅, 刘习奎, 黄光速, 吴锦荣

摘要 动态交联材料通常在水、酸和碱性环境中失去自愈能力和 机械稳定性, 因为这些因素会破坏动态相互作用和动态键. 本文设 计了一种具有疏水外层的胶束状结构来保护离子间的相互作用, 这种结构使离子交联弹性体在水、酸和碱性环境中具有自愈能力 和长期稳定性. 此外, 该弹性体的拉伸强度为 $6.7 \mathrm{MPa}$, 断裂应变为 $1400 \%$, 优于现有的防水自愈弹性体. 结合这些优点, 基于弹性体的 应变传感器和介电弹性体致动器即使在极端恶劣的环境中也具有 高度稳定性和自修复性. 针对动态相互作用设计疏水保护的策略 是非常普遍的, 因此可以推广到其他自愈材料. 